\title{
Composición e invarianza factorial del cuestionario Medida de la Intencionalidad para ser Físicamente Activo (MIFA) en adolescentes mexicanos
}

\section{Composition and factorial invariance of the Measurement of Intention to be Physically Active (MIFA) questionnaire among Mexican adolescents}

\author{
Estrutura e invariancia fatorial do questionario Medida de \\ intencionalidade a ser fisicamente ativa (MIFA) em adolescentes \\ mexicanos
}

\begin{abstract}
Aguirre, J. F.1, Blanco, L. H.2, Mayorga-Vega, D.3, Jurado, P. J.1, Benavides, E. V.1, Ornelas, M.1 1Facultad de Ciencias de la Cultura Física, Universidad Autónoma de Chihuahua, México; $20 P D$ Hospital Civil de Guadalajara, Universidad de Guadalajara; 3Departamento de Didáctica de la Expresión Musical, Plástica y Corporal, Universidad de Jaén
\end{abstract}

\begin{abstract}
RESUMEN
El objetivo de esta investigación fue analizar las propiedades psicométricas propuestos por Moreno, Moreno y Cervelló (2007) para el cuestionario Medida de la Intencionalidad para ser Físicamente Activo (MIFA). La muestra total fue de 549 adolescentes mexicanos 256 mujeres y 293 hombres, con edades comprendidas entre 11-16 años $(M$ $=12.91 ; D E=0.49)$. La estructura factorial del cuestionario se analizó mediante análisis factoriales confirmatorios. Los análisis, muestran que una estructura unifactorial es viable y adecuada tanto para la muestra total $(G F I=.999$; RMSEA < .001; $C F I=1.000)$ como para las poblaciones de hombres $(G F I=.991 ; R M S E A=.074 ; C F I=.995) \mathrm{y}$ mujeres $(G F I=.995 ; R M S E A=.032 ; C F I=.999)$. La estructura unifactorial, atendiendo a criterios estadísticos y sustantivos, ha mostrado adecuados indicadores de ajuste de fiabilidad y validez. Por otro lado, la estructura factorial, las cargas factoriales y los interceptos se consideran invariantes de acuerdo al género; sin embargo, existen diferencias entre hombres y mujeres para la media de intencionalidad para ser físicamente activo. En conclusión, el MIFA-M puede ser una herramienta útil para avanzar en el estudio de los factores que afectan a la práctica de actividad física.

Palabras clave: validez; fiabilidad; psicometría; evaluación psicológica; motivación.
\end{abstract}




\title{
Aguirre, Blanco, Mayorga-Vega, Jurado, Benavides, Ornelas
}

\begin{abstract}
The aim of this research was to analyze the psychometric properties proposed by Moreno et al. (2007) for the Measurement of Intention to be Physically Active (MIFA) questionnaire. The total sample has been composed by 549 Mexican adolescents, 256 women and 293 mean, with ages from 11 to 16 years $(M=12.91, S D=0.49)$. The factor structure of questionnaire has been analized through the confirmatory factor analysis. This analysis shows that a unifactorial structure is viable and adequate for the total sample $(G F I=.999 ; R M S E A<.001 ; C F I=1.000)$ and the populations of men $(\mathrm{GFI}=.991 ; R M S E A=.074 ; C F I=.995)$ and women $(G F I=.995 ; R M S E A=.032 ; C F I=.999)$. The unifactorial structure, according to statistical and substantive criteria, has shown adequate indicators of reliability and validity adjustment. On the other hand, the factorial structure, the factorial loads and the intercepts are considered invariant according to gender; however, there are differences between men and women for the average intentionality of being physically active. In conclusion, the MIFA-M can be a useful tool to advance in the study of the factors that affect the practice of physical activity.
\end{abstract}

Keywords: validity; reliability; psychometrics; psychological assessment; motivation.

\section{RESUMO}

O objetivo do presente estudo instrumental foi analisar a composição e invariância fatorial por gênero do questionário Medida de Intencionalidade a ser Fisicamente Ativa proposto por Moreno et al. (2007) adaptado ao contexto mexicano (MIFA-M) em adolescentes mexicanos. Um total de 549 adolescentes mexicanos, 256 mulheres e 293 homens, com idades entre 11 e 16 anos $(M=12.91 ; D P=.49)$ completaram o questionário MIFA-M. A estrutura fatorial do questionário foi analisada por análises fatoriais confirmatórias. Os resultados mostraram que uma estrutura unifatorial é viável e adequada tanto para a amostra total $(G F I=.999 ; R M S E A<.001 ; C F I=1.000)$ quanto para as populações de homens $(G F I=.991 ; R M S E A=.074 ; C F I=.995)$ e mulheres. Com base em critérios estatísticos e substantivos, a estrutura unifatorial apresentou indicadores adequados de confiabilidade e validade. Além disso, a estrutura fatorial, as cargas fatoriais e os interceptos eram invariantes de acordo com o gênero. Maiores valores de intenção de ser fisicamente ativo em homens do que em mulheres foram encontrados. Maiores valores de intenção de ser fisicamente ativo em homens do que em mulheres foram encontrados. Em conclusão, o MIFA-M é um instrumento útil para avaliar a intenção de ser fisicamente ativo em adolescentes mexicanos.

Palavras chave: validade; precisão; psicometria; avaliação psicológica; motivação.

\section{INTRODUCCIÓN}

La juventud es un período crucial de la vida ya que los hábitos se establecen durante estos años, lo cual podría influir en el comportamiento y el estado de salud durante la edad adulta (Longmuir, Colley, Wherley y Tremblay, 2014). La práctica habitual de actividad física (AF) de la población es ampliamente aceptada como una cuestión clave de salud pública (Poitras et al., 2016; Reiner, Niermann, Jekauc y Woll, 2013). Por ejemplo, existe una fuerte evidencia científica de que durante la adolescencia la práctica regular de $\mathrm{AF}$ moderada-vigorosa se asocia favorablemente con adiposidad, biomarcadores cardiometabólicos, condición física y salud ósea (Baile, González, Ramírez y Suárez, 2011; Fraguela-Vale, VarelaGarrote y Sanz-Arazuri, 2016; Poitras et al., 2016;
Urrutia, Azpillaga, de Cos y Muñoz, 2010). La Organización Mundial de la Salud (OMS, 2010) recomienda que los adolescentes deberían alcanzar diariamente al menos 60 minutos de AF moderadavigorosa.

En la actualidad la inactividad física ya es considerada uno de los mayores problemas de salud pública a escala mundial (World Health Organization, 2014, 2018). Por ejemplo, en América Latina más del $80 \%$ de los adolescentes no cumplen con la recomendación diaria de AF (WHO, 2014). Por ello, hoy en día promocionar en los jóvenes hábitos saludables de AF representa una prioridad de las políticas de salud pública (WHO, 2018). En este sentido, junto con otros estados miembros de la Organización Mundial de la 


\section{Composición e invarianza factorial del cuestionario MIFA}

Salud, recientemente México acordó una reducción relativa del $15 \%$, para el 2030, en la prevalencia de la inactividad física en los jóvenes (WHO, 2018). En adolescentes la intención de ser físicamente activo ha demostrado ser un importante predictor psicológico de los niveles habituales de AF (Sterdt, Liersch y Walter, 2013). Además, basados en diferentes teorías como por ejemplo la Teoría del Comportamiento Planeado (Ajzen, 1985) o el Modelo Trans-contextual (Hagger y Chatzisarantis, 2016), existe evidencia científica de que en adolescentes la intención de ser físicamente activo es la variable más inmediata de la AF habitual (Hagger y Chatzisarantis, 2016).

Para medir la intención de ser físicamente activo, Hein, Müür y Koka (2004) diseñaron y validaron el cuestionario "Intention to be Physically Active". Posteriormente, Moreno et al. (2007) adaptaron y validaron al español la versión del cuestionario bajo el nombre de Medida de la Intencionalidad para ser Físicamente Activo (MIFA). Este instrumento compuesto por cinco ítems evalúa la intención del adolescente de ser físicamente activo tras su paso por las diversas instituciones educativas. Resulta de suma importancia comprobar la estructura factorial de un instrumento y la equivalencia psicométrica del mismo en distintos grupos poblacionales (Abalo, Lévy, Rial y Varela, 2006), siendo la cultura y el género los más relevantes (Marsh, Martin y Jackson, 2010; Viciana, Mayorga-Vega, Guijarro-Romero y Blanco, 2017). Sin embargo, de lo que se conoce no hay estudios instrumentales previos que analicen la estructura factorial del cuestionario MIFA propuesta por Moreno et al. (2007) en adolescentes mexicanos, así como su equivalencia psicométrica en varones y mujeres. Consecuentemente, el objetivo de la presente investigación instrumental (Ato, López y Benavente, 2013) fue analizar la composición e invarianza factorial por género del cuestionario MIFA adaptado al contexto mexicano (MIFA-M) en adolescentes mexicanos.

\section{MATERIAL Y MÉTODOS}

\section{Participantes}

Un total de 549 adolescentes del norte de México, 256 mujeres y 293 varones, fueron seleccionados en el presente estudio mediante un muestreo por conveniencia. La edad de los participantes fluctuó entre los 11 y 16 años, con una media de 12.91 años y una desviación estándar de 0.49 años. Todos los participantes cumplimentaron el cuestionario voluntariamente.

\section{Instrumentos}

En el presente estudio se utilizó una versión adaptada al contexto mexicano del cuestionario en español MIFA de Moreno et al. (2007), el cual denominamos MIFA-M. El cuestionario MIFA consta de cinco ítems que pertenecen a un solo constructo que mide la intención del adolescente de ser físicamente activo tras su paso por las diversas instituciones educativas. Con los ítems precedidos de la frase "Respecto a tu intención de practicar alguna actividad físicodeportiva...", los participantes responden de acuerdo a una escala tipo Likert que oscilaba de 1 a 5 , donde 1 corresponde a "totalmente en desacuerdo" y 5 a "totalmente de acuerdo". El cuestionario MIFA ha mostrado propiedades psicométricas adecuadas en adolescentes españoles $(C F I=.98 ; T L I=.96 ; R M S E A$ $=0.056 ;$ SRMR $=.018 ; \alpha=.94)$.

En la adaptación para el presente estudio de la versión en español de Moreno et al. (2007) se hicieron las siguientes tres modificaciones: Para la primera adaptación mientras que la versión original se puntúa con cinco opciones de respuesta, de acuerdo con estudios previos (por ejemplo, Viciana, MayorgaVega, Guijarro-Romero y Martínez-Baena, 2017), en la versión utilizada en la presente investigación los participantes eligieron entre 11 posibles respuestas para que se adaptara a las calificaciones escolares a la que los adolescentes están acostumbrados en el sistema educativo de México. Por tanto, conjugamos la escala cualitativa original con la versión del presente estudio para que quedara de la siguiente manera: "completamente en desacuerdo" (0), "en desacuerdo" (1, 2 y 3), "ni de acuerdo ni en desacuerdo" (4, 5 y 6), "de acuerdo" (7, 8 y 9), y "completamente de acuerdo" (10); La segunda adaptación consistió en cambiar algunos términos utilizados en los ítems de la versión original con el fin de utilizar un lenguaje más adecuado al contexto de la cultura mexicana (por ejemplo, la frase "después de terminar el instituto" por "después de terminar la secundaria"), y la tercera adaptación consistió en aplicar el instrumento por medio de una computadora con el fin de permitir el almacenamiento de los datos sin etapas previas de codificación, con una mayor precisión y rapidez. 


\section{Aguirre, Blanco, Mayorga-Vega, Jurado, Benavides, Ornelas}

\section{Procedimiento}

Antes de comenzar con el estudio se informó y solicitó permiso a las autoridades educativas de centros educativos de Educación Secundaria de la ciudad de Chihuahua (México). Posteriormente, se informó a las familias y estudiantes, así como que se les invitó a participar. Las familias y estudiantes que aceptaron participar firmaron el consentimiento/ asentimiento informado. Posteriormente se aplicó el cuestionario MIFA-M mediante medios informáticos en las propias aulas de los centros educativos en una sesión de aproximadamente 15 minutos. Al inicio de cada sesión se hizo una pequeña introducción sobre los objetivos y la importancia de la investigación, así de cómo acceder al instrumento. Se les solicitó la máxima sinceridad y se les garantizó la confidencialidad de los datos que se obtuvieran. Las instrucciones de cómo responder se encontraban en las primeras pantallas, antes del primer ítem del instrumento. Al término de la sesión se les agradeció su participación. Una vez aplicado el instrumento se procedió a recopilar y a analizar los datos mediante el paquete SPSS $18.0 \mathrm{y}$ AMOS 21.0.

\section{Análisis estadístico}

Para obtener un instrumento que presente las mejores propiedades psicométricas en adolescentes varones y mujeres, el análisis psicométrico del MIFA-M se realizó en dos etapas: (1) Análisis de las propiedades psicométricas del instrumento, y (2) Análisis de invarianza factorial.

Análisis de las propiedades psicométricas del instrumento. El primer paso del análisis de las propiedades psicométricas del cuestionario consistió en calcular la asimetría y la curtosis de cada ítem, para determinar si se cumplía el supuesto de normalidad. En seguida se sometieron a comparación dos modelos de medida: el MIFA-M1, que responde a una estructura unifactorial acorde a la distribución original de los ítems dentro del cuestionario y el MIFA-M1f, que corresponde a la estructura factorial del modelo anterior sin el ítem 1 de más baja saturación en el factor. Posteriormente, se calculó la fiabilidad del factor del mejor de los modelos de medida obtenido mediante el Coeficiente de Alfa de Cronbach (Elosua y Zumbo, 2008; Nunnally y Bernstein, 1995) y del Coeficiente Omega (Revelle y Zinbarg, 2009; Sijtsma, 2009).
Análisis de invarianza factorial. Con el fin de obtener una prueba que presente las mejores propiedades para la conformación de los puntajes del cuestionario MIFA-M en varones y mujeres adolescentes se llevó a cabo un análisis de la invarianza factorial, tomando como base el mejor modelo obtenido en la muestra total (es decir, modelo MIFA-M1f). Posteriormente se calculó la fiabilidad, en ambas muestras a través del Alfa de Cronbach y el Coeficiente Omega (Revelle y Zinbarg, 2009).

Los análisis factoriales confirmatorios se realizaron mediante el software AMOS 21 (Arbuckle, 2012). Las varianzas de los términos de error fueron especificadas como parámetros libres y en la variable latente (factor) uno de los coeficientes estructurales se igualo a uno para que la escala del factor fuera igual a la de las variables observables (ítems). El método de estimación empleado fue el de Máxima Verosimilitud (ML) con la aplicación de procedimientos de remuestreo bootstrap para los casos de no normalidad (Byrne, 2016); aunque en AMOS 21.0 el ML es especialmente robusto para posibles casos de no normalidad, especialmente si la muestra es suficientemente amplia y los valores de asimetría y curtosis no son extremos (asimetría $<|2|$ y curtosis < |7|). Además, siguiendo la recomendación de Thompson (2004), cuando se emplea el análisis factorial confirmatorio se debe corroborar no sólo el ajuste de un modelo teórico sino que es recomendable comparar los índices de ajuste de varios modelos alternativos para seleccionar el mejor.

Para evaluar el ajuste del modelo se emplearon el estadístico Chi-cuadrado, el índice de bondad de ajuste (GFI) y el error cuadrático medio de aproximación (RMSEA) como medidas absolutas de ajuste. El índice de bondad ajustado (AGFI), el Índice Tucker-Lewis (TLI) y el índice de bondad de ajuste comparativo (CFI) como medidas de ajuste incremental. La razón de Chi-cuadrado sobre los grados de libertad (CMIN/GL) y el Criterio de Información de Akaike (AIC) como medidas de ajuste de parsimonia (Byrne, 2016; Gelabert et al., 2011). La literatura previa considera aceptables valores para estos índices como sigue: GFI y CFI $\geq$.90; RMSEA por debajo de .08; y CMIN/GL menor a 5 (Hu y Bentler, 1999). 


\section{Composición e invarianza factorial del cuestionario MIFA}

\section{RESULTADOS}

Asimetría y curtosis de los ítems del cuestionario

En la Tabla 1, además, de las medias y desviaciones estándar, se resumen los valores de asimetría y curtosis de las distintas variables contempladas en el modelo de medida. La mayoría de las variables muestran valores de asimetría de $\pm 1.50 \mathrm{y} \pm 1.00$ de curtosis, por lo que se infiere que las variables se ajustan razonablemente a una distribución normal; por otro lado el índice multivariado de Mardia por debajo del valor 70 indica normalidad multivariada (Rodríguez y Ruiz, 2008).

Tabla 1

Asimetría y curtosis de los ítems del cuestionario MIFA-M. Muestra total.

\begin{tabular}{|c|c|c|c|c|}
\hline Ítem & $\bar{M}$ & $\mathrm{DE}$ & AS & $\overline{\mathrm{CU}}$ \\
\hline$\overline{\text { Ítem } 1}$ & 7.58 & 2.74 & -1.08 & 0.40 \\
\hline Ítem 2 & 8.09 & 2.59 & -1.42 & 1.40 \\
\hline Ítem 3 & 7.20 & 3.18 & -0.97 & -0.15 \\
\hline Ítem 4 & 7.93 & 2.71 & -1.34 & 1.03 \\
\hline Ítem 5 & 7.00 & 3.34 & -0.91 & -0.42 \\
\hline índice multivariado de Mardia & & & & 24.33 \\
\hline
\end{tabular}

Nota: $\mathrm{M}=$ media, $\mathrm{DE}=$ desviación estándar, $\mathrm{AS}$ = asimetría; $\mathrm{CU}$ = curtosis

\section{Análisis factorial confirmatorio muestra total}

Los resultados globales del análisis factorial confirmatorio $(\mathrm{GFI}=.990 ; \mathrm{RMSEA}=.058 ; \mathrm{CFI}$ = .993) para el modelo MIFA-M1 que corresponde a una estructura unifactorial acorde a la distribución original de los ítems dentro del cuestionario, indican que el modelo de medición es óptimo (Tabla 2). El factor del modelo MIFA-M1 explica aproximadamente el $62 \%$ de la varianza y de acuerdo a los resultados obtenidos 4 de los 5 ítems saturan por encima de .60 en su dimensión prevista (Tabla 3).

Tabla 2

Índices absolutos, incrementales y de parsimonia para los modelos generados.

\begin{tabular}{|c|c|c|c|c|c|c|c|c|}
\hline \multirow[b]{2}{*}{ Modelo } & \multicolumn{3}{|c|}{ Índices absolutos } & \multicolumn{3}{|c|}{ Índices incrementales } & \multicolumn{2}{|c|}{ Índices de parsimonia } \\
\hline & $\chi^{2}$ & GFI & RMSEA & AGFI & TLI & $\mathrm{CFI}$ & CMIN/DF & AIC \\
\hline MIFA-M1 & $14.118^{*}$ & .990 & .058 & .969 & .985 & .993 & 2.824 & 34.118 \\
\hline MIFA-M1f & 0.831 & .999 & .000 & .996 & 1.000 & 1.000 & 0.416 & 16.831 \\
\hline
\end{tabular}

Nota: $* \mathrm{p}<.05$

Los resultados globales del análisis factorial confirmatorio $(\mathrm{GFI}=.999$; $\mathrm{RMSEA}<.001$; $\mathrm{CFI}=$ 1.000) del segundo modelo sometido a prueba (MIFAM1f) que corresponde a la estructura factorial del modelo anterior sin el ítem 1 de más baja saturación en el factor, indican que este modelo de medición es mejor que el modelo anterior y que su ajuste es óptimo (Tabla 2). El factor de este modelo explica aproximadamente el $72 \%$ de la varianza. Por otro lado los cuatro ítems saturan por encima de .60 en su dimensión prevista (Tabla 3). El factor del modelo MIFA-M1, obtiene valores de consistencia interna de $\alpha=.841$ y $\Omega=.854$ con una varianza media extraída $(A V E)=.551 \mathrm{y}$ el factor del modelo MIFA-M1f, obtiene valores de consistencia interna de $\alpha=.864 \mathrm{y}$ $\Omega=.874$ con una $A V E=.637$. 


\section{Aguirre, Blanco, Mayorga-Vega, Jurado, Benavides, Ornelas}

Tabla 3

Soluciones estandarizadas análisis factorial confirmatorio para los Modelos MIFA-M1 y MIFA-MIf.

\begin{tabular}{lcc}
\hline \multicolumn{1}{c}{ Ítem } & MIFA-M1 & MIFA-M1f \\
\hline 1. Me interesa el desarrollo de mi forma física, para no estar débil, mediante la práctica de deporte & .44 & - \\
2. Además de las clases de Educación Física, me gusta practicar deporte & .76 & .75 \\
3. Después de terminar la secundaria, quisiera formar parte de un club deportivo & .83 & .83 \\
4. Después de terminar la secundaria, me gustaría mantenerme activo practicando un deporte & .92 & .92 \\
5. Habitualmente practico deporte en mi tiempo libre & .67 & .67 \\
\hline
\end{tabular}

\section{Análisis factoriales confirmatorios hombres y mujeres}

Tanto en la muestra de hombres como de mujeres todas las variables muestran valores de asimetría de \pm 1.60 y \pm 1.00 de curtosis, además, el índice multivariado de Mardia por debajo del valor 70 indica normalidad multivariada (Rodríguez y Ruiz, 2008).

De acuerdo a los resultados de la Tabla 4 el análisis factorial confirmatorio de 4 ítems agrupados en un solo factor en la muestra de mujeres fue óptimo ( $G F I$ $=.995 ; R M S E A=.032$ ) y de acuerdo a las medidas de ajuste incremental y de parsimonia significativamente superior al modelo independiente y muy similar al modelo saturado.

Por otro lado, el análisis factorial confirmatorio en la muestra de hombres (Tabla 4), indica nuevamente que el modelo de medición unifactorial era óptimo ( $G F I$ $=.991 ; R M S E A=.074) \mathrm{y}$ de acuerdo a las medidas de ajuste incremental y de parsimonia significativamente superior al modelo independiente y muy similar al modelo saturado. De acuerdo a los resultados de la Tabla 5, en ambas muestras, la mayoría de los ítems saturan por encima de .70 en su dimensión (factor) prevista.

Tabla 4

Índices absolutos, incrementales y de parsimonia para los modelos generados. Análisis factorial confirmatorio para mujeres y hombres.

\begin{tabular}{|c|c|c|c|c|c|c|c|c|}
\hline \multirow[b]{2}{*}{ Modelo } & \multicolumn{3}{|c|}{ Índices absolutos } & \multicolumn{3}{|c|}{ Índices incrementales } & \multicolumn{2}{|c|}{ Índices de parsimonia } \\
\hline & $\chi^{2}$ & GFI & RMSEA & AGFI & TLI & CFI & CMIN/DF & AIC \\
\hline \multicolumn{9}{|c|}{ Solución factorial para las mujeres } \\
\hline $\begin{array}{l}1 \text { factor } 4 \\
\text { ítems }\end{array}$ & 2.534 & .995 & .032 & .975 & .997 & .999 & 1.267 & 18.534 \\
\hline Saturado & 0.000 & 1.000 & & & 1.000 & 1.000 & & 20.000 \\
\hline Independiente & $511.784 *$ & .479 & .575 & .132 & .000 & .000 & 85.297 & 519.784 \\
\hline \multicolumn{9}{|c|}{ Solución factorial para los hombres } \\
\hline $\begin{array}{l}1 \text { factor } 4 \\
\text { ítems }\end{array}$ & 5.226 & .991 & .074 & .995 & .985 & .995 & 2.613 & 21.226 \\
\hline Saturado & .000 & 1.000 & & & & 1.000 & & 20.000 \\
\hline Independiente & $664.806^{*}$ & .432 & 613 & .053 & .000 & .000 & 110.801 & 672.806 \\
\hline
\end{tabular}

Nota: $* \mathrm{p}<.05$ 


\section{Composición e invarianza factorial del cuestionario MIFA}

Tabla 5

Soluciones estandarizadas para el análisis factorial confirmatorio en ambas muestras.

\begin{tabular}{lcc}
\hline \multicolumn{1}{c}{ Ítem } & Mujeres & Hombres \\
\hline 2. Además de las clases de Educación Física, me gusta practicar deporte & .71 & .78 \\
3. Después de terminar la secundaria, quisiera formar parte de un club deportivo & .87 & .79 \\
4. Después de terminar la secundaria, me gustaría mantenerme activo practicando un deporte & .92 & .93 \\
5. Habitualmente practico deporte en mi tiempo libre & .58 & .76 \\
\hline
\end{tabular}

\section{Invarianza de la estructura factorial entre mujeres y hombres}

Los índices de ajuste obtenidos (Tabla 6) permitieron aceptar la equivalencia de los modelos de medida básicos entre las dos muestras. El valor de Chicuadrado está por debajo al exigido para aceptar la hipótesis de invarianza, los índices $G F I=.993, C F I$ $=.997, R M S E A=.041$ y $A I C=39.761$ refuerzan esta conclusión lo que permite aceptar el modelo base de la invarianza (modelo sin restricciones).

Añadiendo al modelo base restricciones sobre las cargas factoriales caracterizamos la invarianza métrica. Los valores que se recogen en la Tabla 6 permiten aceptar este nivel de invarianza. El índice de ajuste general $(G F I=.986)$ y el error cuadrático medio de aproximación $(R M S E A=.048)$ siguen aportando información convergente en el sentido de la invarianza métrica. Además, el criterio de información de Akaike $(A I C=41.945)$ y el índice comparativo de Bentler $(C F I=.992)$ no sufren grandes variaciones respecto al modelo anterior. Haciendo uso del criterio para la evaluación de los modelos anidados propuesto por Cheung y Rensvold (2002), quiénes sugieren que si el cálculo de la diferencia de los CFI de ambos modelos anidados disminuye en $.01 \mathrm{o}$ menos, se debe de dar por bueno el modelo restringido $\mathrm{y}$, por tanto, el cumplimiento de la invarianza factorial. En este sentido, la diferencia entre CFIs obtenida permitió aceptar el modelo de invarianza métrica. Se pude concluir que las cargas factoriales fueron equivalentes en las dos muestras

Una vez demostrada la invarianza métrica entre las muestras, pasamos a evaluar la equivalencia entre interceptos (invarianza factorial fuerte). Los índices (Tabla 6) mostraron un ajuste óptimo de este modelo, tanto evaluado de modo independiente como analizándolo respecto a su anidamiento con el modelo de invarianza métrica. La diferencia entre los índices comparativos de Bentler fue menor a una milésima; el índice de ajuste general es .985 y el error cuadrático medio de aproximación es .045. Aceptada la invarianza fuerte, los dos modelos evaluados son equivalentes respecto a los coeficientes factoriales y a los interceptos. El factor del modelo MIFA-M en la muestra de mujeres, obtiene valores de consistencia interna de $\alpha=.843$ y $\Omega=.859$ con una varianza media extraída $(A V E)=.611$ y en la muestra de hombres, obtiene valores de consistencia interna de $\alpha=.881$ y $\Omega=.889$ con una $A V E=.669$.

Tabla 6

Índices de bondad de ajuste de cada uno de los modelos puestos a prueba en la invarianza factorial.

\begin{tabular}{lcccccccc}
\hline \multicolumn{1}{c}{ Modelo } & \multicolumn{7}{c}{ Índice de Ajuste } \\
\cline { 2 - 8 } & $\chi^{2}$ & $\mathrm{gl}$ & GFI & NFI & CFI & RMSEA & AIC \\
\hline $\begin{array}{l}\text { Modelo sin } \\
\text { restricciones }\end{array}$ & 7.761 & 4 & .993 & .993 & .997 & .041 & 39.761 \\
$\begin{array}{l}\text { Invarianza métrica } \\
\text { Invarianza }\end{array}$ & $15.945^{*}$ & 7 & .986 & .986 & .992 & .048 & 41.945 \\
factorial fuerte & $16.807^{*}$ & 8 & .985 & .986 & .992 & .045 & 40.807 \\
$\begin{array}{l}\text { Nota: } * \text { p < .05; GFI = índice de bondad de ajuste; NFI = índice de ajuste } \\
\text { normado; CFI = índice de ajuste comparativo; RMSEA = error cuadrático } \\
\text { medio de aproximación; AIC = criterio de Información de Akaike }\end{array}$
\end{tabular}

\section{Contraste de las medias entre mujeres y hombres}

Una vez comprobada la invarianza factorial, las diferencias entre las medias del factor de los dos grupos se estimaron tomando como referente la muestra de hombres, fijando en 0 el valor de las medias para dicha muestra y estimando libremente el valor de las medias para la muestra de mujeres. Las restricciones sobre los coeficientes de regresión e interceptos, requeridos para los contrastes entre las medias se realizaron automáticamente mediante el software AMOS 21 (Arbuckle, 2012). Los resultados de las comparaciones entre medias indicaron que las mujeres, en comparación con los hombres, presentan 


\section{Aguirre, Blanco, Mayorga-Vega, Jurado, Benavides, Ornelas}

una menor intencionalidad para ser físicamente activas $(-.487, p<0.05)$.

\section{DISCUSIÓN Y CONCLUSIONES}

El objetivo del presente estudio instrumental fue analizar la composición e invarianza factorial por género del cuestionario MIFA adaptado al contexto mexicano (MIFA-M) en adolescentes mexicanos. Además, de la estructura factorial de un instrumento, es necesario evaluar si la misma estructura factorial es aplicable a distintas poblaciones (Abalo et al., 2006). En este sentido, los resultados del presente estudio mostraron que para el MIFA-M la estructura de un factor es fiable y válida en adolescentes mexicanos, tanto para la muestra total como para los varones y mujeres separadamente.

Los análisis factoriales confirmatorios realizados en la muestra total apoyan la estructura factorial de un solo factor. El factor así obtenido presenta en general saturaciones factoriales estandarizadas adecuadas, saturaciones que se corresponden con la estructura propuesta para el cuestionario por Moreno et al. (2007). De manera similar, en una muestra de 988 adolescentes españoles de 15-17 años, Moreno et al. (2007) encontraron adecuados resultados para un estructura unifactorial con los cinco ítems $(\mathrm{CFI}=.98$; TLI $=.96 ;$ RMSEA $=.056 ;$ SRMR $=.018)$. No obstante, en el presente estudio cuando se eliminaba el ítem 1 ("Me interesa el desarrollo de mi forma física, para no estar débil, mediante la práctica de deporte") por su baja saturación, el modelo fue significativamente mejor.

El análisis factorial confirmatorio tanto en la muestra de mujeres como de varones, indicó que el ajuste de los datos al modelo teórico de 4 ítems agrupados en un solo factor es óptimo. Asimismo, el factor así obtenido presenta saturaciones factoriales estandarizadas adecuadas. Lamentablemente, no se encontraron estudios previos que analizaran la varianza factorial del MIFA. Por otro lado, el factor en ambas muestras también evidenció una consistencia interna muy adecuada, especialmente si se considera el número reducido de ítems (Revelle y Zinbarg, 2009). De manera similar, Moreno et al. (2007) con la muestra de adolescentes españoles obtuvieron una consistencia interna igual a .94.
Conjuntamente con todo lo antes dicho, los resultados del análisis de la invarianza factorial entre varones y mujeres indican una alta congruencia entre pares de factores. Dicho resultado sugiere la existencia de fuertes evidencias de la validación cruzada de la medida y, por tanto, de la estabilidad de la estructura (Abalo et al., 2006). Por otro lado, las comparaciones entre los grupos reflejaron diferencias significativas a favor de los varones en cuanto su intencionalidad para ser físicamente activos. En esta línea, numerosos estudios previos con adolescentes han encontrado diferencias estadísticamente significativas a favor de los varones tanto en la intención de ser físicamente activo (Martínez-Baena y Mayorga-Vega, 2014) como de la conducta activa (Mayorga-Vega, Parra y Viciana, 2017).

En cuanto a las limitaciones del presente estudio, la primera se relaciona con el hecho de que por cuestiones prácticas no se pudo realizar un muestreo representativo de los adolescentes mexicanos, lo cual limita la posibilidad de generalizar los hallazgos del presente estudio a toda la población. Por ello, estudio futuros debería analizar la propiedades psicométricas del MIFA-M con una muestra representativa de adolescentes mexicanos. En segundo lugar la invarianza factorial solo se analizó en función del género. En este sentido, estudios futuros deberían comprobar si la invarianza de la estructura de la escala se cumple por otras características relevantes de la población como, por ejemplo, el grupo de edad o la práctica deportiva. Por último, cabe destacar que es indispensable que estudios futuros comprueben si el cuestionario resulta útil para predecir la adherencia hacia el inicio y el mantenimiento de la conducta activa. La realización de dichas investigaciones futuras permitirían confirmar la estructura obtenida, lo cual permitirá contar con evidencia más robusta respecto a la estructura factorial de la escala.

En conclusión, el análisis de las propiedades psicométricas del cuestionario MIFA-M ha mostrado que una estructura unifactorial es viable, adecuada e invariante entre varones y mujeres adolescentes mexicanos. Atendiendo a criterios estadísticos y sustantivos, la estructura unifactorial ha mostrado adecuados indicadores de ajuste, fiabilidad y validez. 


\section{Composición e invarianza factorial del cuestionario MIFA}

\section{APLICACIONES PRÁCTICAS}

Potenciar en los jóvenes la intencionalidad para ser físicamente activos sin duda contribuye a su salud y calidad de vida, de ahí la necesidad de contar con instrumentos fiables y válidos para su medición. Por ello, el presente estudio analiza las propiedades psicométricas de una adaptación al contexto mexicano (MIFA-M) del cuestionario MIFA propuestos por Moreno et al. (2007). Este estudio además sirve de premisa para futuras investigaciones sobre instrumentos para la medición de la intencionalidad para ser físicamente activo en poblaciones con diferentes factores personales y culturales. Finalmente, el presente instrumento será de gran utilidad para la aplicación en diferentes ámbitos como, por ejemplo, estudios descriptivos o de intervención.

\section{AGRADECIMIENTOS}

La Secretaría de Educación Pública-Subsecretaría de Educación Superior-Dirección de Superación Académica- Programa para el Desarrollo Profesional Docente (DE-13 -6894) quién financió este estudio. Así como la facilidad para la financiación de la publicación (CONACYT, Redes Temáticas, No. 269614).

\section{REFERENCIAS}

1. Abalo, J., Lévy, J., Rial, A. y Varela, J. (2006). Invarianza factorial con muestras múltiples. En J. Lévy (Ed.), Modelización con Estructuras de Covarianzas en Ciencias Sociales (pp. 259-278). Madrid: Netbiblo.

2. Ajzen, I. (1985). From Intentions to Actions: A Theory of Planned Behavior. En J. Kuhl \& J. Beckmann (Eds.), Action Control: From Cognition to Behavior (pp. 11-39). Heidelberg: Springer.

3. Arbuckle, J. R. (2012). AMOS users guide version 21.0. Chicago, IL: Marketing Department, SPSS Incorporated.

4. Ato, M., López, J. J. y Benavente, A. (2013). Un sistema de clasificación de los diseños de investigación en psicología. Anales de Psicología, 29(3), 1038-1059.

5. Baile, J. I., González, A., Ramírez, C. y Suárez, P. (2011). Imagen corporal, hábitos alimentarios y hábitos de ejercicio físico en hombres usuarios de gimnasio y hombres universitarios no usuarios. Revista de Psicología del Deporte, 20(2), 353366.

6. Byrne, B. M. (2016). Structural Equation Modeling With AMOS: Basic Concepts, Applications, and Programming. New York, NY: Routledge.

7. Cheung, G. W. y Rensvold, R. B. (2002). Evaluating goodness-of-fit indexes for testing measurement invariance. Structural Equation Modeling, 9(2), 233-255. doi: 10.1207/s15328007SEM0902_5

8. Elosua, P. y Zumbo, B. D. (2008). Coeficientes de fiabilidad para escalas de respuesta categórica ordenadas. Psicothema, 20(4), 896-901.

9. Fraguela-Vale, R., Varela-Garrote, L. y SanzArazuri, E. (2016). Ocio deportivo, imagen corporal y satisfacción vital en jóvenes españoles. Revista de Psicología del Deporte, 25(2), 33-38.

10. Gelabert, E., García-Esteve, L., Martín-Santos, R., Gutiérrez, F., Torres, A. y Subirà, S. (2011). Psychometric properties of the Spanish version of the Frost Multidimensional Perfectionism Scale in women. Psicothema, 23(1), 133-139.

11. Hagger, M. S. y Chatzisarantis, N. L. D. (2016). The Trans-Contextual Model of Autonomous Motivation in Education: Conceptual and Empirical Issues and Meta-Analysis. Review of Educational Research, 86(2), 360-407. doi: 10.3102/0034654315585005

12. Hein, V., Müür, M. y Koka, A. (2004). Intention to be Physically Active after School Graduation and Its Relationship to Three Types of Intrinsic Motivation. European Physical Education Review, $\quad 10(1), \quad 5-19 . \quad$ doi: 10.1177/1356336x04040618

13. Hu L., y Bentler P. M. (1999). Cutoff criteria for fit indexes in covariance structure analysis: Conventional criteria versus new alternatives. Structural Equation Modeling, 6(1), 1-55. doi: 10.1080/10705519909540118

14. Longmuir, P., Colley, R., Wherley, V. y Tremblay, M. (2014). Risks and benefits of childhood physical activity. The Lancet Diabetes and Endocrinology, 2(11), 861-862. doi: 10.1016/S2213-8587(14)70221-9 


\section{Aguirre, Blanco, Mayorga-Vega, Jurado, Benavides, Ornelas}

15. Marsh, H. W., Martin, A. J. y Jackson, S. (2010). Introducing a Short Version of the Physical Self Description Questionnaire: new strategies, shortform evaluative criteria, and applications of factor analyses. Journal of Sport \& Exercise Psychology, 32(4), 438-482.

16. Martínez-Baena, A. C. y Mayorga-Vega, D. (2014). Intención de ser físicamente activos en escolares granadinos: Diferencias entre chicos y chicas. Revista Internacional de Deportes Colectivos, 7(18), 74-75.

17. Mayorga-Vega, D., Parra, M. y Viciana, J. (2017). Comparison of moderate-to-vigorous physical activity levels between physical education, school recess and after-school time in secondary school students. Kinesiology, 49(2), 242-251. doi: 796.012.13:613-053.6

18. Moreno, J. A., Moreno, R. y Cervelló, E. (2007). El autoconcepto físico como predictor de la intención de ser físicamente activo. Psicología y Salud, 17(2), 261-267.

19. Nunnally, J. C. y Bernstein, I. H. (1995). Teoría Psicométrica. México: McGraw-Hill.

20. Organización Mundial de la Salud. (2010). Recomendaciones mundiales sobre actividad física para la salud. Suiza: Organización Mundial de la Salud.

21. Poitras, V. J., Gray, C. E., Borghese, M. M., Carson, V., Chaput, J.-P., Janssen, I., . . . Tremblay, M. S. (2016). Systematic review of the relationships between objectively measured physical activity and health indicators in schoolaged children and youth. Applied Physiology, Nutrition, and Metabolism, 41(6), 197-239. doi: 10.1139/apnm-2015-0663

22. Reiner, M., Niermann, C., Jekauc, D. y Woll, A. (2013). Long-term health benefits of physical activity-a systematic review of longitudinal studies. BMC public health, 13(1), 813-822. doi: 10.1186/1471-2458-13-813

23. Revelle, W. y Zinbarg, R. E. (2009). Coefficients alpha, beta, omega and the glb: comments on Sijtsma. Psychometrika, 74(1), 145-154. doi: 10.1007/s11336-008-9102-z

24. Rodríguez, M. N. y Ruiz, M. A. (2008). Atenuación de la asimetría y de la curtosis de las puntuaciones observadas mediante transformaciones de variables: Incidencia sobre la estructura factorial. Psicológica, 29, 205-227.

25. Sijtsma, K. (2009). On the use, the misuse, and the very limited usefulness of Cronbach's alpha. Psychometrika, 74(1), 107-120. doi: 10.1007/s11336-008-9101-0

26. Sterdt, E., Liersch, S. y Walter, U. (2013). Correlates of physical activity of children and adolescents: A systematic review of reviews. Health Education Journal, 73(1), 72-89. doi: $10.1177 / 0017896912469578$

27. Thompson, B. (2004). Exploratory and Confirmatory Factor Analysis. Understanding concepts and applications. . Washington, D C: American Psychological Association.

28. Urrutia, S., Azpillaga, I., de Cos, G. L. y Muñoz, D. (2010). Relación entre la percepción de estado de salud con la práctica físicodeportiva y la imagen corporal en adolescentes. Cuadernos de Psicología del Deporte, 20 (2), 51-56.

29. Viciana, J., Mayorga-Vega, D., Guijarro-Romero, S. y Blanco, H. (2017). The Spanish Adaptation of the Sport Motivation Scale-II in Adolescent Athletes. Psychological Reports, 120(5), 943-695.

30. Viciana, J., Mayorga-Vega, D., Guijarro-Romero, S. y Martínez-Baena, A. C. (2017). Effect of two alternated teaching units of invasion team sports on the tactical learning in primary schoolchildren. International Journal of Performance Analysis in Sport, 17(3), 256-270. doi: 10.1080/24748668.2017.1331575

31. World Health Organization. (2014). Global status report on noncommunicable diseases 2014. Switzerland: World Health Organization.

32. World Health Organization. (2018). Global action plan on physical activity 2018-2030: more active people for a healthier world. Switzerland: World Health Organization. 